\title{
Article \\ Sedimentation of Two Side-by-Side Heavy Particles of Different Density in a Shear-Thinning Fluid with Viscoelastic Properties
}

\author{
Sensen Yang ${ }^{1}$, Chengxu Tu ${ }^{1,2,3, *}$, Minglu Dai ${ }^{1}$, Xianfu Ge ${ }^{4}$, Rongjun Xu ${ }^{1}$, Xiaoyan Gao ${ }^{1}$ and Fubing Bao ${ }^{1, *}$ \\ 1 Zhejiang Provincial Key Laboratory of Flow Measurement Technology, China Jiliang University, \\ Hangzhou 310018, China; s1902080446@cjlu.edu.cn (S.Y.); s20020804009@cjlu.edu.cn (M.D.); \\ xurongjun628@163.com (R.X.); gaoxy_star@cjlu.edu.cn (X.G.) \\ 2 College of Control Science and Engineering, Zhejiang University, Hangzhou 310027, China \\ 3 LEO Group Co., Ltd., Wenling 317500, China \\ 4 Zhejiang Machinery \& Electrical Group Co., Ltd., Hangzhou 310002, China; gexf@zj926.com \\ * Correspondence: tuchengxu@cjlu.edu.cn (C.T.); dingobao@cjlu.edu.cn (F.B.)
}

Citation: Yang, S.; Tu, C.; Dai, M.; Ge, X.; Xu, R.; Gao, X.; Bao, F.

Sedimentation of Two Side-by-Side Heavy Particles of Different Density in a Shear-Thinning Fluid with Viscoelastic Properties. Appl. Sci. 2021, 11, 7113. https://doi.org/ 10.3390/app11157113

Academic Editor: Francesca Scargiali

Received: 1 July 2021

Accepted: 29 July 2021

Published: 31 July 2021

Publisher's Note: MDPI stays neutral with regard to jurisdictional claims in published maps and institutional affiliations.

Copyright: () 2021 by the authors. Licensee MDPI, Basel, Switzerland. This article is an open access article distributed under the terms and conditions of the Creative Commons Attribution (CC BY) license (https:// creativecommons.org/licenses/by/ $4.0 /)$.

\begin{abstract}
Particle sedimentation has widely existed in nature and engineering fields, and most carrier fluids are non-Newtonian. Recently, the manipulation of a settling particle in liquid has been a topic of high interest to those involved in engineered processes such as composite materials, pharmaceutical manufacture, chemistry and the petroleum industry. Compared with Newtonian fluid, the viscosity of non-Newtonian fluid is closely related to the shear rate, leading to a single settling particle having different dynamic behaviors. In this article, the trajectories and velocities of two side-by-side particles of different densities (heavy and light) settling in a shear-thinning fluid with viscoelastic property were studied, as well as that for the corresponding single settling particle. Regardless of the difference in the particle density, the results show the two-way coupling interaction between the two side-by-side settling particles. As opposed to a single settling particle, the wake of the heavier particle can clearly attract or rebound the light particle due to the shear-thinning or viscoelastic property of the fluid. Regarding the trajectories of the light particle, three basic path types were found: (i) the light particle is first attracted and then repelled by the wake of the heavy one; (ii) the light particle approaches and then largely traces within the path of the heavy one in the limited field of view; (iii) the light particle is first slightly shifted away from its original position and then returns to this initial position. In addition to this, due to the existence of a corridor of reduced viscosity and negative wake generated by the viscoelastic property, the settling velocity of a light particle can exceed the terminal velocity of a single particle of the same density. On the other hand, the sedimentation of the light particle can induce the distinguishable transverse migration of the heavy one.
\end{abstract}

Keywords: particle sedimentation; two side-by-side particles; viscoelastic fluid; shear-thinning fluid

\section{Introduction}

Particle sedimentation can be found in a wide range of natural and industrial processes, such as river flows, blood flows, microfluidic chips and fluidization etc. [1-5]. The settling particle is strongly affected by the rheological properties of surrounding fluid as well as the physical properties of the particle. Knowledge on particle behaviors in Newtonian liquids is relatively extensive. Magnaudet [6] summarized the forces that act on spherical particles, which usually include steady drag, history force, added mass effects and lift force. In addition, it is possible to predict interactions between dispersed particles moving in Newtonian fluids [7]. Clift proposed that when the particle Reynolds number is large $\left(750<\operatorname{Re}<2 \times 10^{5}\right)$, the viscous resistance of the particle is negligible compared with the inertial force [5]. Considering the relationship between the Reynolds number $(R e)$ and 
the mass ratio $\left(m^{*}\right)$, three typical sedimentation trajectories of a single particle settling in glycerin-water mixture were found: vertical, oblique and zigzag [8], where $\left(m^{*}\right)$ is the relative density of the particle compared to the fluid, a map of regimes of the particle trajectories is presented for $R e=100-12,000$ and $m^{*}=0-1.5$. Five types of trajectories of a free-settling particle in Newtonian fluids have been found: steady and oblique, oblique and oscillating regime with low frequency, oblique and oscillation regime with high frequency, zigzagging periodic regime and three-dimensional chaotic regime [9]. The settling velocities of dual particles would be larger than a single particle at small Reynolds numbers [10]. It has been widely confirmed that the drag coefficient of a particle settling in Newtonian fluids only depends on Re. In the case of two particles, based on a numerical study using the three-dimensional lattice Boltzmann method, Nie and Lin [11] identified six sedimentation patterns for two side-by-side particles of different densities settling in a Newtonian fluid. They also simulated two particles of different densities released in tandem [12]. As for the theoretical analysis, the asymptotic forms of the far-field and near-field for the particle velocities were given by Wacholder and Sather [13]. In this work, the two particles were unequal in size and density, and both the inertia of the particles and the fluid can be neglected, as opposed to with the viscous forces.

Since many processes involving particle settling occur in non-Newtonian fluids [14,15], such as hydraulic fracture for oil exploration [16], sedimentation velocity analytical ultracentrifugation in biopharmaceuticals [17], the behavior of particles in the human respiratory tract [18] and the transport of the particles in microfluidic chips [19], understanding particle settling behavior in non-Newtonian fluids is of significant importance to the above applications. However, this is much more complex for particles moving in non-Newtonian fluids. Specifically, the viscosity of the fluid around the particle changes with the variation of the distribution of shear stress caused by the settling particle, which finally results in a change in the drag coefficient [7]. Thus, the conclusions drawn from the investigation of Newtonian fluid are not appropriate for non-Newtonian fluids. Various experimental and numerical studies have been carried out on the sedimentation of particles in different non-Newtonian fluids, such as power-law fluids [20], Carreau fluids [21], and Boger fluids [22,23]. Particles can behave in completely different ways in non-Newtonian fluids due to the coupling effects of viscoelasticity and shear-dependent viscosity. For example, obvious negative wakes would occur when particle settling in different viscoelastic fluids. The increase in the elasticity would cause the negative wakes to move closer to the particle [24]. The pioneering studies on the two particles settling in viscoelastic fluids were performed by Riddle et al. [25], Joseph et al. [26], and Bot et al. [27]. A critical distance which distinguishes between the attraction and repulsion of a pair of particles settling in water-glycerin mixture of polyacrylamide was revealed by Riddle et al. [25]. A particular range of the initial particle-particle distance, within which inter-particle attraction of two side-by-side settling particles was observed, was given by Joseph et al. [26]. They considered the first normal stress difference to be the explanation for particle-particle attraction. As particles settled in a shear-thinning fluid with a thixotropic and viscoelastic effect, three types of particle interaction, strong particle-particle attraction, weak particle-particle attraction and repulsion were observed [28]. For two identical particles released in tandem in viscoelastic fluid, two settling modes were observed numerically: (i) a dipole would emerged as the trailing particle approaching the leading one; (ii) a stable terminal distance between the particles was established [29]. The two settling modes have also been recently confirmed by the experimental results reported by Freire et al. [30], in which shear thinning fluid with a viscoelastic effect was adopted, and which modes occurred depended on the initial distance and the fluid viscosity.

In this paper, the sedimentation of a single particle in a shear-thinning viscoelastic fluid was first examined for comparison. Then, we investigated the settling behavior of two side-by-side particles with different densities and focused on the effect of the initial particle-particle distance and the density difference on their interaction. 


\section{Experimental Method}

The experimental setup was mainly composed of a transparent tank, a particle releasing system and a shadowgraph setup, as shown in Figure 1. The tank is an open acrylic container with a height of $1000 \mathrm{~mm}$ with a squared cross-section with a 500-mm width, and is filled with polyacrylamide solution. The two side-by-side particles arranged horizontally can be released simultaneously with a specified distance as required by the particle releasing system, which uses an ejector to generate the vacuum pressure in the two particle pipettes. We used two high-speed cameras (FASTCAM Mini UX, Photron, Yonezawa, Japan), two LED arrays and a computer linked to the cameras to facilitate high-speed shadowgraph binocular-imaging. Thus, the particle trajectories can be tracked on both $x-z$ and $y-z$ planes through this binocular imaging system. Meanwhile, we used the particle image velocimetry (PIV) system to investigate the flow field in the wake of the particle (Figure 2). The PIV system includes a high-speed camera (FASTCAM Mini UX, Photron, Yonezawa, Japan), a continuum laser (SN: CL007, Beijing SMHY Technology Co. Ltd., Beijing, China), a computer linked to the camera and the fluorescent tracer particles (W-TG1, Beijing HSKT Technology Co. Ltd., Beijing, China). Three types of spherical particles (with less than a $0.25 \mu \mathrm{m}$ diameter variation and spherical error and $0.02 \mu \mathrm{m}$ of the surface roughness) were used in our experiments (Table 1), and $0.5 \mathrm{wt} \%$ polyacrylamide (PAAm, supplied by Shanghai Macklin Biochemical Co., Shanghai, China) solution was used. The density of PAAm solution is $1005 \mathrm{~kg} / \mathrm{m}^{3}$, which is very close to that of water. The rheological properties of the PAAm solution were examined at $20^{\circ} \mathrm{C}$ by a rheometer (MCR 702 Multidrive, Anton Paar Co., Graz, Austria). Figure 3 show the rheological curves of the shear stress $\tau$ to the shear rate $\gamma$ with steady state in the PAAm solution and pure water, where the slope of the curves represents the fluid viscosity $\eta$. Figure 4 shows the rheological curves of the fluid viscosity $\eta$ to the shear rate $\gamma$ in the PAAm solution and pure water. It can be seen that the shear stress $\tau$ and shear rate $\gamma$ of pure water have a linear relationship, while PAAm solution exhibits a very high viscosity value at a low shear rate $\gamma$. With the increase of the shear rate $\gamma$, the viscosity of PAAm solution decreases and becomes stable. This indicates that the PAAm solution has a strong shear thinning property [31].

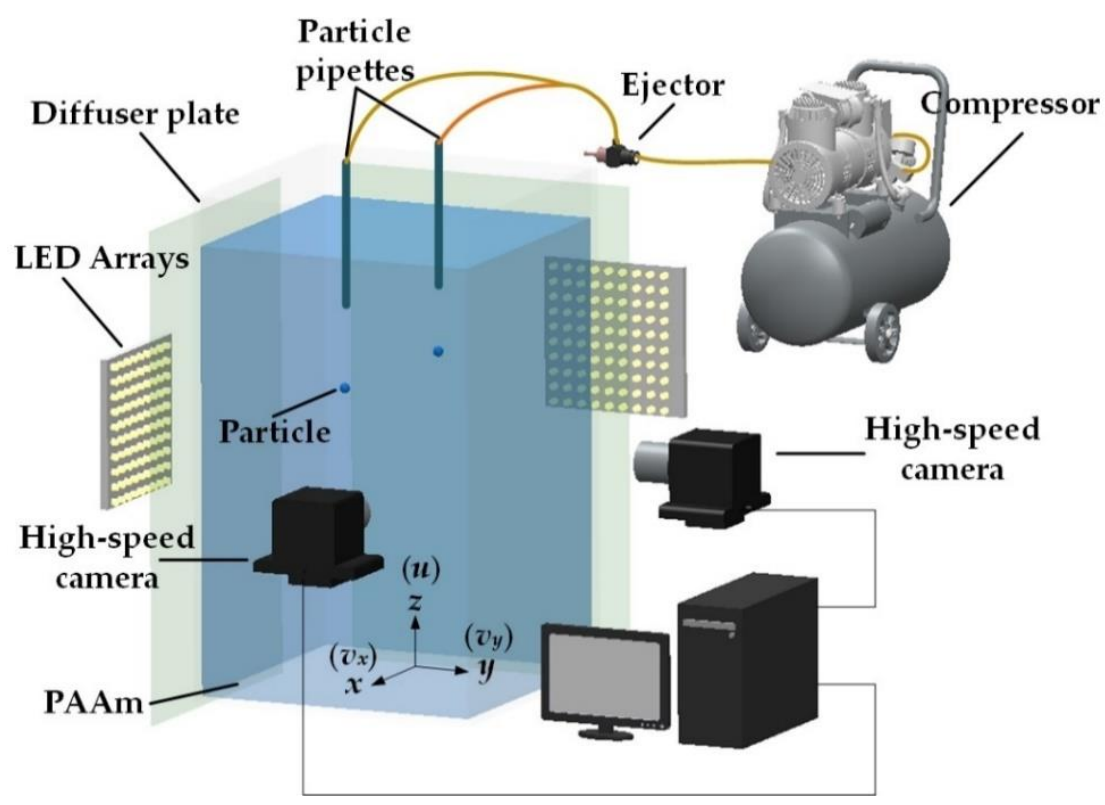

Figure 1. Schematic of the experimental setup for two side-by-side particles settling. 


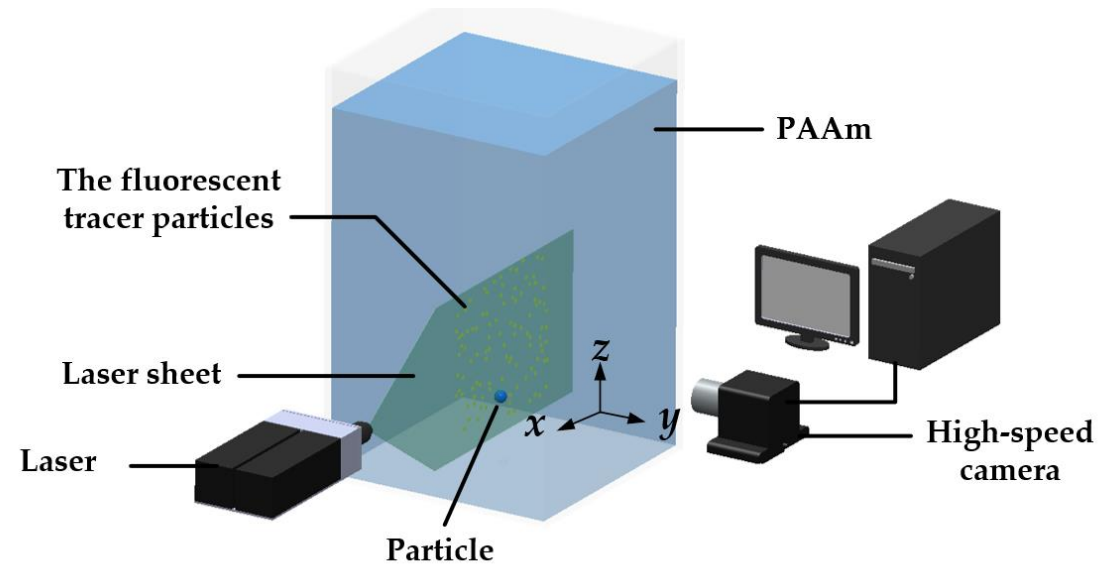

Figure 2. Schematic of the PIV system for investigating the flow field of the wake behind the particle.

Table 1. Properties of spherical particles.

\begin{tabular}{|c|c|c|}
\hline Material & Density $\left(\mathrm{kg} / \mathrm{m}^{3}\right)$ & Diameter (mm) \\
\hline Silicon nitride ceramics $\left(\mathrm{Si}_{3} \mathrm{~N}_{4}\right)$ & 3200 & 9.525 \\
\hline Zirconia ceramics $\left(\mathrm{ZrO}_{2}\right)$ & 6000 & 9.525 \\
\hline Stainless steel & 7930 & 9.525 \\
\hline
\end{tabular}

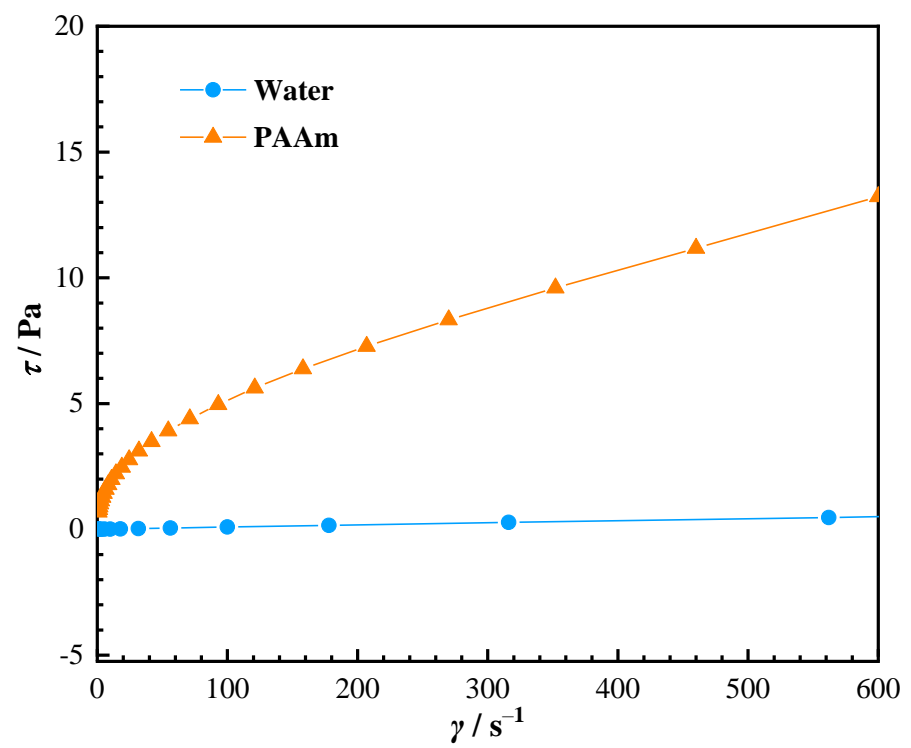

Figure 3. Rheogram of $0.5 \mathrm{wt} \%$ PAAm solution.

In order to verify whether the PAAm solution has viscoelasticity, the frequency sweep response was performed through the MCR rheometer at a shear strain $=1 \%$. The data (Figure 5) indicate that the storage modulus $G^{\prime}$ and loss modulus $G^{\prime \prime}$ increased with the increasing frequency, which indicates the yield behavior of the test solution. This demonstrates that the PAAm solution in this study is a viscoelastic fluid by Liu et al. [32]. Apart from this, the negative wake (the instantaneous profile) was confirmed by our results using PIV (Figure 6). In the previous work, the negative wake was considered a large strain phenomenon associated with viscoelasticity $[33,34]$ and the reason for this formation was proven to derive from the shear and extensional motion of the particle wake in the viscoelastic fluid. Under the complex shear and extensional motion, the viscoelastic fluid exhibits rapid elastic recoil, similar to an elastic thread, which seems to be the cause of the negative wake $[33,34]$. Thus, it can be concluded that the PAAm solution in this study is a viscoelastic fluid. 


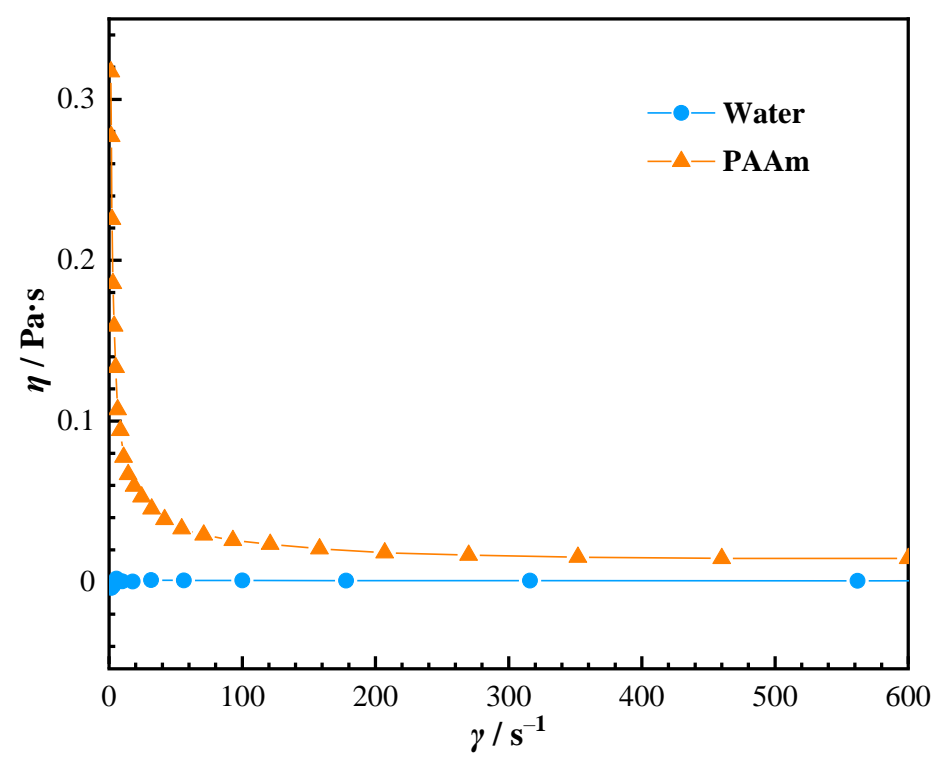

Figure 4. Dynamic viscosity for the $0.5 \mathrm{wt} \%$ PAAm solution as a function of the shear rate.

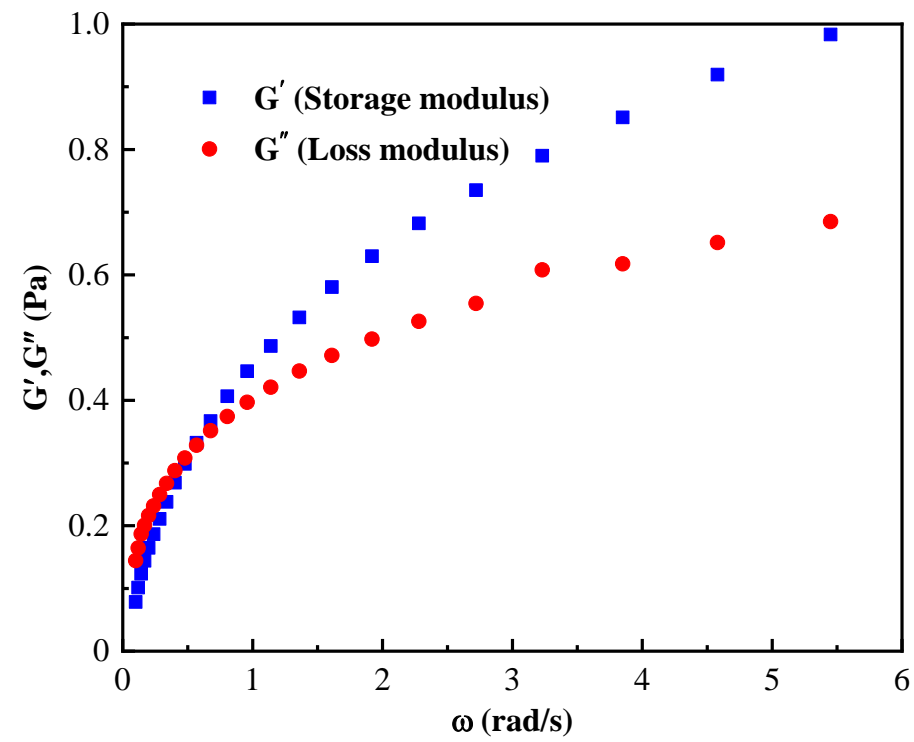

Figure 5. Viscoelastic modulus of PAAm solution as a function of frequency.

For comparison, the settling behavior of a single particle and two particles were both detected using the binocular imaging system at a frame rate of $1000 \mathrm{fps}$. In order to prevent the gas film or bubbles from adhering on the particle surface during particle sedimentation, the particles were immersed in PAAm solution preliminarily several times.

In our experiments, both the initial particle-particle distance and the density difference between the two particles were varied, but the particle size remained similar. The dimensionless distance, $L^{*}$, was adopted to describe the initial horizontal distance between the two particles (Figure 7), given as

$$
L^{*}=L / d
$$

where $L$ is the initial center distance between the two particles, and $d$ is the particle diameter $(d=9.525 \mathrm{~mm})$. 


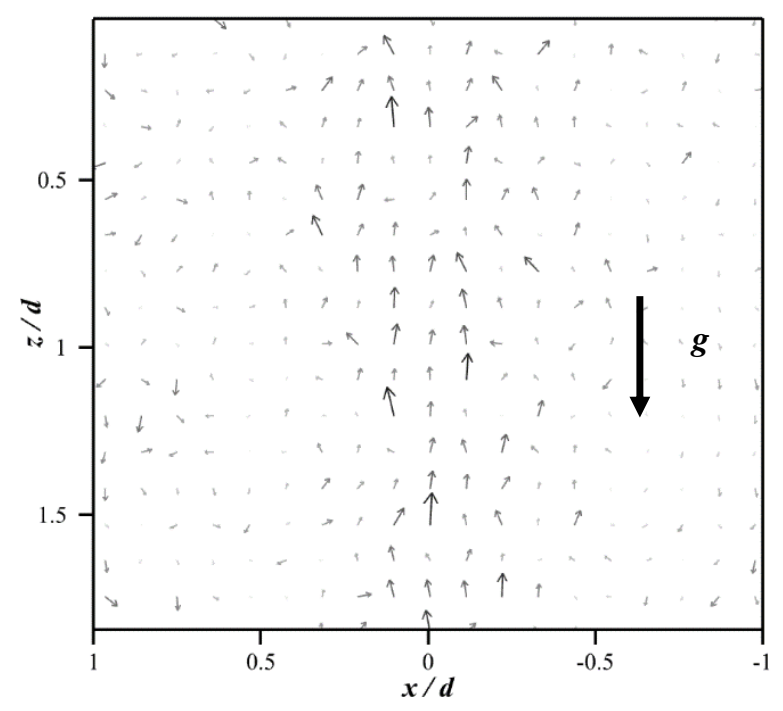

Figure 6. The negative wake at $7 d$ downstream from the particle center.

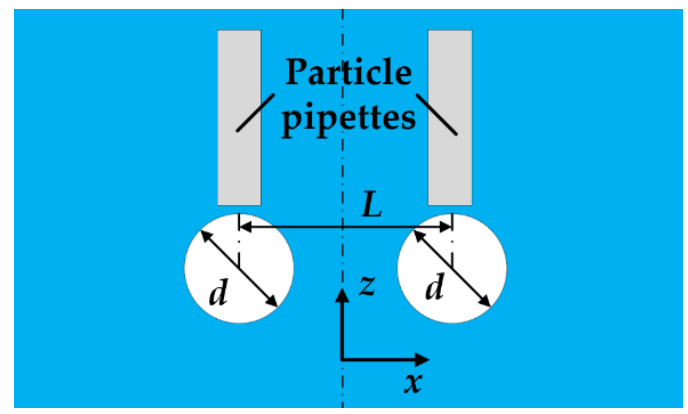

Figure 7. Schematic of the sedimentation of two side-by-side particles.

Additionally, parameter $\alpha$ is introduced to denote the density difference between the two particles and is given by [35]

$$
\alpha=\frac{\rho_{p H}-\rho_{p L}}{\rho_{l}}
$$

where $\rho_{p L}, \rho_{p H}$ are the densities of light particle and heavy particle, respectively; $\rho_{l}$ is the density of fluid. The different $\alpha$ values used in our experiments are listed, i.e., $\alpha=1.92$ ( $\mathrm{ZrO}_{2}$ and Stainless steel), $2.79\left(\mathrm{Si}_{3} \mathrm{~N}_{4}\right.$ and $\left.\mathrm{ZrO}_{2}\right)$ and $4.71\left(\mathrm{Si}_{3} \mathrm{~N}_{4}\right.$ and Stainless steel).

In our experiments, there were several sources of experimental error of the spherical particles such as the diameter variation, spherical error and the surface roughness. Three types of spherical particles with a diameter variation less than $0.25 \mu \mathrm{m}$ and spherical error and a surface roughness $0.02 \mu \mathrm{m}$ were used in our experiments. Compared with the $d$ $(9.525 \mathrm{~mm})$, the errors of the spherical particles can be ignored.

Meanwhile, in order to improve the sensitivity and synchronization of the control particles in the experiment, we designed a new synchronous particle release device, which uses the vacuum suction principle of the ejector (Figure 1). This device can precisely control the release timing and spatial location of the particles and ensure the simultaneous release and of the two side-by-side particles at the same horizontal level. The particles settling experiments under each condition are performed at least $4 \sim 10$ times to ensure the recurrence. The particle settling path in the PAAm solution was remarkably stable and the experimental results have good repeatability. After each set of test experiment, an interval period of $1 \mathrm{~h}$ was required to restore fluid stability. 


\section{Results and Discussion}

\subsection{Force Analysis}

The forces acting on the moving particles in a quiescent fluid have been widely studied $[5,7,36]$. When the moving particles reach the terminal velocity, the particles are in a force equilibrium. During sedimentation, the particles are mainly subject to gravity $G$, and buoyancy $F_{B}$, and the total drag force $F_{D}$ (Figure 8).

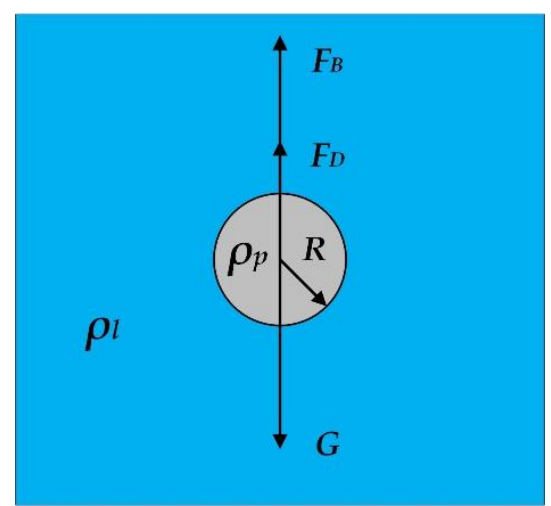

Figure 8. Schematic of the forces acting on the particle.

The forces acting on the particle in Figure 8 can be expressed as

$$
G=F_{B}+F_{D}
$$

The gravity on the particles can be expressed as

$$
G=m g=\frac{1}{6} \pi d^{3} \rho_{p} g
$$

And the buoyancy of the particles during settling:

$$
F_{B}=V \rho_{l} g=\frac{1}{6} \pi d^{3} \rho_{l g}
$$

where $d$ is the particle diameter, $\rho_{p}$ is the particle density, and $\rho_{l}$ is the fluid density.

In non-Newtonian fluids, the power law model, which has been used widely in previous work to describe the fluids' rheology, has the following form [37]:

$$
\tau=k \gamma^{n}
$$

where $k$ is the consistency index and $n$ is the power law index.

Meanwhile, $n$ and $k$ are the power law constants in a power law fluid. For a Rheogram of $0.5 \mathrm{wt} \%$ PAAm solution (Figure 3), we can determine that the value of $n$ is 0.484 and the value of $k$ is 0.572 (Goodness of fit $=0.997$ ) by the curve fitting equation (Equation (6)).

A modified particle Reynolds number was defined for the settling particle in the shear thinning fluid [38]:

$$
R e^{*}=\frac{d^{n} u_{p}^{2-n} \rho_{l}}{k}
$$

where $u_{p}$ is the sedimentation velocity of particles.

The total drag force of the particle settling in a power law fluid was given by [39]

$$
F_{D}=3 \pi k d^{2}\left(\frac{u_{p}}{d}\right)^{n} f(n)
$$

where $f(n)$ is a function of the power law constant. 
From the above equation, we can obtain the following:

$$
f(n)=\frac{d^{n+1} g\left(\rho_{p}-\rho_{l}\right)}{18 k u_{p}{ }^{n}}
$$

In our experiments, the terminal velocities of single particle settling in the PAAm solution are $1.44 \mathrm{~m} / \mathrm{s}$ (for stainless steel), $1.24 \mathrm{~m} / \mathrm{s}$ (for $\mathrm{ZrO}_{2}$ ), and $0.77 \mathrm{~m} / \mathrm{s}$ (for $\mathrm{Si}_{3} \mathrm{~N}_{4}$ ), respectively.

Therefore, the values of $f(n)$ are 5.539 (for stainless steel), 4.295 (for $\mathrm{ZrO}_{2}$ ), and 2.377 (for $\mathrm{Si}_{3} \mathrm{~N}_{4}$ ), respectively.

Apart from this, the values of $\mathrm{Re}^{*}$ are 321 (for stainless steel), 256 (for $\mathrm{ZrO}_{2}$ ), and 124 (for $\mathrm{Si}_{3} \mathrm{~N}_{4}$ ), respectively.

\subsection{Sedimentation of a Single Particle}

In this section, the behavior of a single settling solid spherical particle in the PAAm solution is presented, which was used to compare with that of two side-by-side particles in Section 3.3.

In the previous work, we obtained the settling velocity of the corresponding particles in water [40]. The results show that the sedimentation velocity in the initial acceleration stage is basically proportional to the time for $t<0.2 \mathrm{~s}$, regardless of the particle density. After this linear acceleration stage, all the particles in water achieved their terminal velocities at $t \approx 0.4 \mathrm{~s}$.

Figure 9 shows the settling behaviors of a single particle in the PAAm solution with different particle densities. It is clear that for all cases the particle motion in both $x-z$ and $y-z$ planes is almost straight and the transverse motion can be neglected. In other words, the particle settling path can be regarded as a vertical line in the PAAm solution. This phenomenon can be caused by the viscoelastic property of PAAm solution, the fluidization boundary between the settling particles and the viscoelastic fluid acts as a cylinder wall $[24,28]$. Therefore, the friction generated by the fluidization boundary position in relation to the settling particle surface results in a sharp decline in the transverse velocity of the particle settling [41]. Thus, the transverse migration for a single particle can be neglected and the settling path can be regarded as a vertical line. In contrast, particles settling in water usually exhibit a three-dimensional unstable path $[36,40]$ due to the unsteady trajectory and the wake turning to three-dimensional and unsteady behind the settling particle.
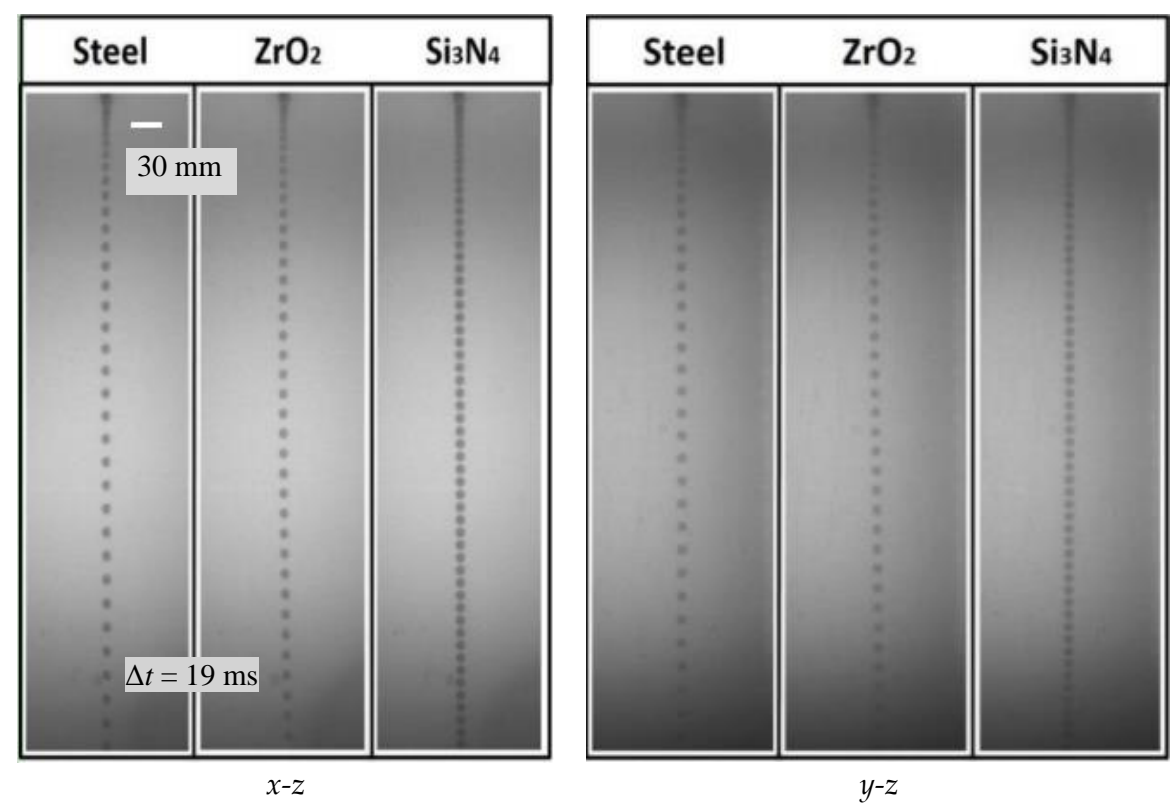

Figure 9. Settling behaviors of single particle. The time interval between adjacent particles is $19 \mathrm{~ms}$. 
In addition, Figure 10 shows the variation of the particle velocity and the normalized trajectory in PAAm solution for different particles. The migration velocity is decomposed into the transverse velocities $v_{x}$ in $x-z$ plane, $v_{y}$ in $y$ - $z$ plane and the vertical velocity $u$. The transverse migration is normalized by the particle diameter and denoted as $s^{*}$, which is also plotted as a function of time in Figure 10. The very small $s^{*}$ quantitatively reflects that there is no obvious transverse motion of the settling particles. This can also be confirmed from the near-zero transverse velocities during the whole testing process.

In our experiments, before reaching the terminal velocity, the particle would experience a short acceleration leading to an increase in the vertical velocity, while this acceleration hardly changes the transverse velocity in the viscoelastic fluid.

In PAAm solution, although the difference in particle density leads to a difference in the terminal settling velocity, the particles almost achieved their terminal velocities at $t \approx 0.4 \mathrm{~s}$. As shown in the Figure 11, the terminal settling velocity of the particle $\left(\mathrm{Si}_{3} \mathrm{~N}_{4}\right)$ in PAAm solution is lower than that in water. Apart from this, the terminal velocities of other particles (stainless steel and $\mathrm{ZrO}_{2}$ ) are higher than that in water, and the deviation of terminal velocity from that of the particle settling in water increases with the particle density. Based on this, we speculate that there is a critical value of $R e^{*}$, i.e., another particle with a different density, and its terminal settling velocity in the PAAm solution is consistent with that in water. The reason why the terminal settling velocity of the particle $\left(\mathrm{Si}_{3} \mathrm{~N}_{4}\right)$ in the PAAm solution is lower in water but not the other two particles is the relatively weak shear thinning effect. The modified particle Reynolds number (321 (for stainless steel), 256 (for $\mathrm{ZrO}_{2}$ ), and 124 (for $\mathrm{Si}_{3} \mathrm{~N}_{4}$ )) was mentioned for the settling particle in the PAAm solution. Moreover, it has been confirmed that the terminal settling velocity increases with the increasing $R e^{*}$ in the shear thinning fluids in the previous work [36], which can also be observed in Figure 11. We can find that the $\operatorname{Re}^{*}\left(\mathrm{Si}_{3} \mathrm{~N}_{4}\right)$ is smaller than that in the other two particles, which indicates that the viscous resistance occupies a more dominant role than the initial resistance in the particle $\left(\mathrm{Si}_{3} \mathrm{~N}_{4}\right)$ sedimentation [36]. Thus, the terminal settling velocity of the particle $\left(\mathrm{Si}_{3} \mathrm{~N}_{4}\right)$ in the PAAm solution is slower and the shear thinning effect is relatively weak. As shown in the Figure 11, the error bar of the $u$ in the PAAm solution represents the standard error of a set of the parallel experiments. Notably, the $u$ of $\mathrm{Si}_{3} \mathrm{~N}_{4}$ particle settling in water fluctuates at $t=0.2 \mathrm{~s}$, which is probably caused by the unsteady trajectory and the wake becoming three-dimensional and unsteady behind the settling particle $[5,7]$, but this phenomenon disappeared due to the obvious viscoelastic property of the PAAm solution.

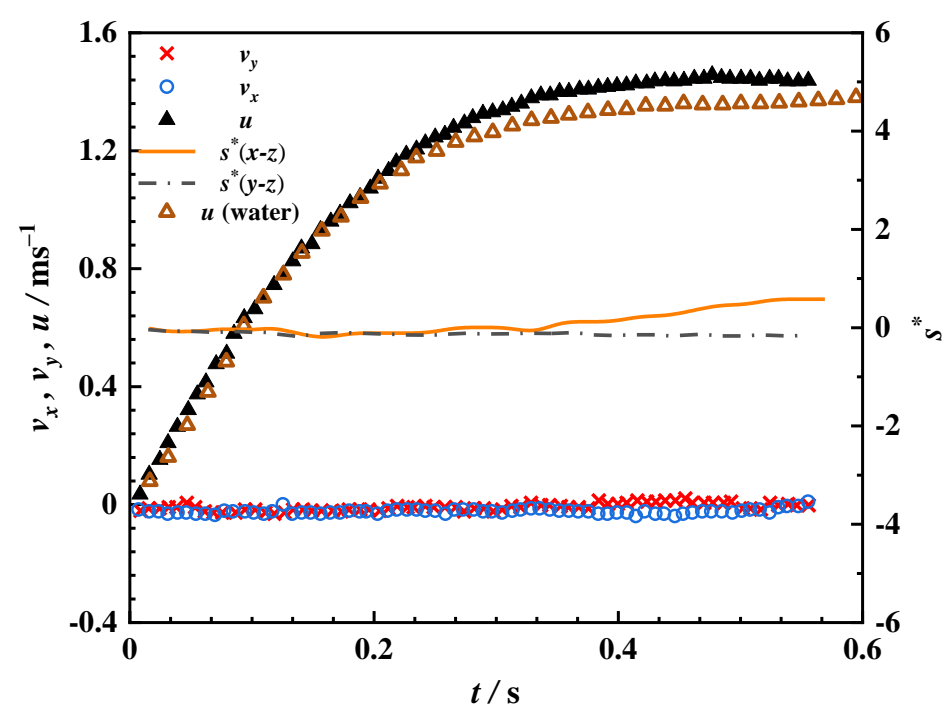

(a) Stainless steel

Figure 10. Cont. 


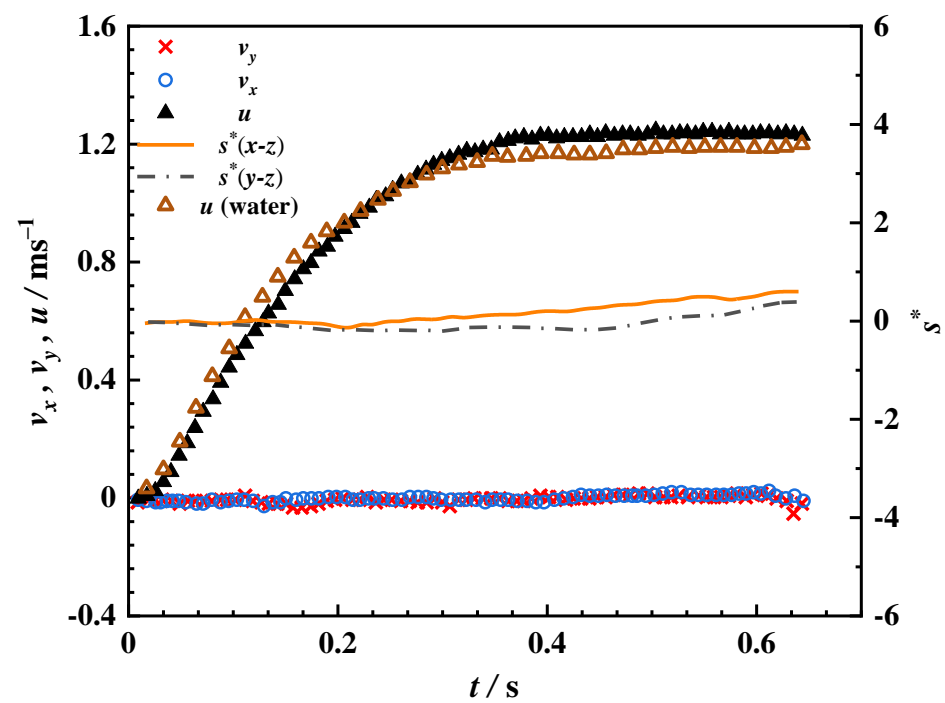

(b) $\mathrm{ZrO}_{2}$

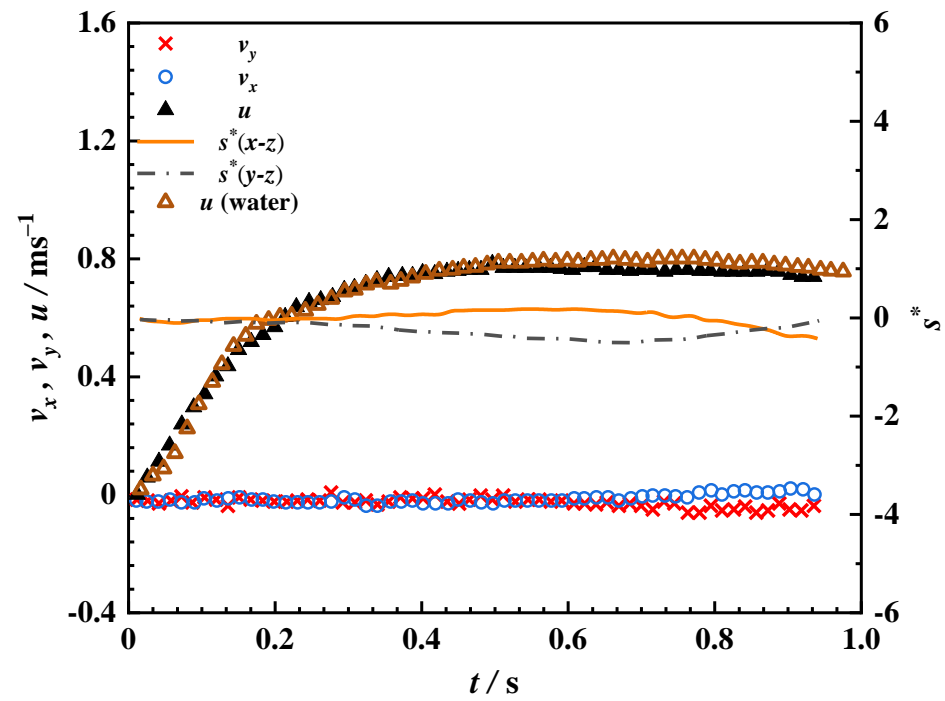

(c) $\mathrm{Si}_{3} \mathrm{~N}_{4}$

Figure 10. Variations in the trajectories and velocity of a single particle settling in PAAm solution. $v_{x}$ is the transverse velocity in $x-z$ plane, $v_{y}$ is the transverse velocity in $y-z$ plane, $u$ is the vertical velocity.

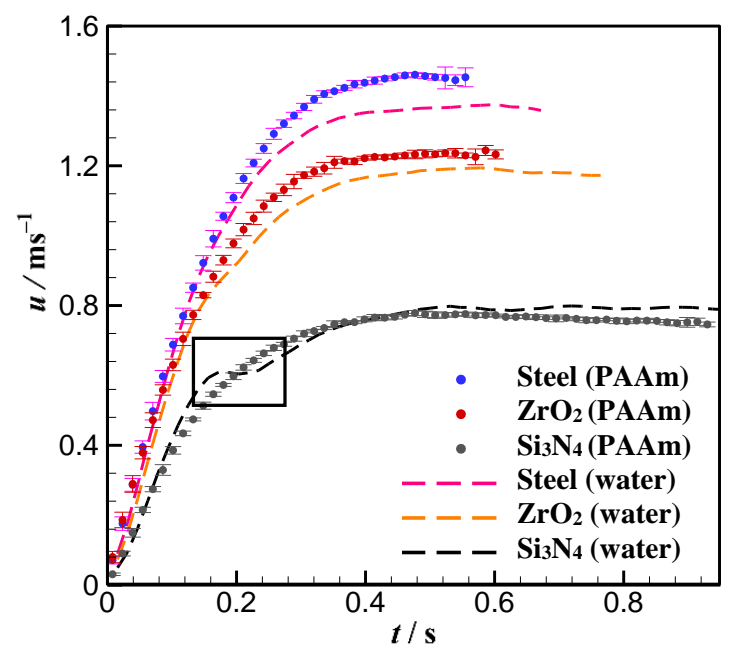

Figure 11. Variation in vertical velocity $u$ of a single particle with different densities in PAAm solution and water. 


\subsection{Trajectory of Side-by-Side Particles of Different Densities}

As discussed above, the path of a single particle settling in PAAm solution can be approximately regarded as a vertical line. In comparison, for the sedimentation of two similar side-by-side particles, a particle-particle interaction would occur and cause the particles to deviate from the vertical path at small $L^{*}$ [7]. This interaction and deviation from the case of a single particle can be enhanced by the identical particle terminal velocity, which results in a highly similar elevation of the two particles. Therefore, within the critical distance $L^{*}$, the drafting-kissing-tumbling phenomenon (DKT) had been widely observed for this initial particle configuration $[7,28]$.

In our study, we found that the difference of particles' density leads to great difference in settling velocity (Figure 12), and then the vertical distance between the particles is enlarged with time. Nevertheless, the wake of the heavy particle, in a leading position, can still affect the light particle settling into a non-linear path. The particle-particle interaction can also cause the transverse migration of the heavy particle, and the smaller the $\alpha$, the greater the transverse migration. Notably, at the low value of $\alpha$, the path of the heavy particle settling can be approximately regarded as a vertical line at $L^{*}<3$. The clear transverse migration of the heavy particle occurred at $3<L^{*}<6.1$ and disappeared at $L^{*}>7.1$.

Compared to the trajectory of heavy particles, the particle-particle interaction has a stronger impact on the light particle. There are three basic types of trajectories of light particles in the $x-z$ plane as the light particle traces the heavy one:

Type (i) in the horizontal direction $(x)$, the light particle first is strongly attracted and then is repelled by the rectilinear path of the heavy one (for $L^{*}=1.2$ and $\alpha=1.92,2.79$ and 4.71).

Type (ii) the light particle approaches the heavy one in the $x$ direction, and then travel a certain distance within the path of the heavy one (for $L^{*}=2.0$ and $\alpha=1.92$ and 2.79) without bouncing from the wake of the heavy particle in the limited field of view of the camera.

Type (iii) the light particle is slightly away from the path of the heavy one at first in the horizontal direction, and then the light particle moves back to its original position in the $x$ direction (for $L^{*}=3$ and $\alpha=1.92,2.79$ and 4.71)

Figure 13 shows three basic types of settling trajectories of the side-by-side particles. The line connecting the particles illustrates the instantaneous position of this pair of particles at a certain moment.

In the cases of type (i), two particles settle vertically at first, and the heavy particle travels faster due to its larger density, causing the appearance of a corridor with reduced viscosity (CRV) due to the shear-thinning property of the fluid. Then, the light particle deviated from its initial position and was attracted by this corridor in the $x$ direction. This phenomenon is similar to the drafting stage of the DKT pattern in the settling of two particle with different densities in a Newtonian fluid [11] and the attracting behavior reported in two particles settling in viscoelastic fluids $[7,20,28]$. In Newtonian fluids, the approach of two particle is mostly due to the lower pressure caused by the wake of the leading particle [42]. While in viscoelastic fluids (such as PAAm solution), a widely accepted explanation for particle-particle attraction is that the first normal stress difference of the viscoelastic fluid modifies the pressure distribution on the particle surface [7]. Hence in the current experiments, the viscoelasticity of the PAAm solution could have caused the light particle to approach the heavy one in the $x$ direction. Further, the light particle would be sucked into the corridor with a reduced viscosity behind the heavy particle. The particleparticle collision that has been reported in the settling of two identical particles in shear thinning liquids $[20,28]$ was not observed in our experiments as a result of the different sedimentation velocities caused by the different densities. The heavier particle's settling velocity is larger than the lighter one; thus the lighter particle is only attracted to the CRV. Interestingly, after a certain distance following the heavy particle's wake, the light particle would rebound from the CRV and the horizontal distance between the particles increases gradually. Since the PAAm solution is a shearing thinning viscoelastic fluid, the fluid will deform under stress due to the particle motion and partial recovery to the undeformed 
state once the stress disappears [7]. Inspired by this, the negative wake behind the settling particles was successfully captured in our experiments, as shown in Figure 6. The negative wake probably induced the downstream fluid and the particle to move upwards. Therefore, the combined effect of the shear-thinning property of the PAAm solution and the negative wake could be responsible for the type (i) path of the light particle.
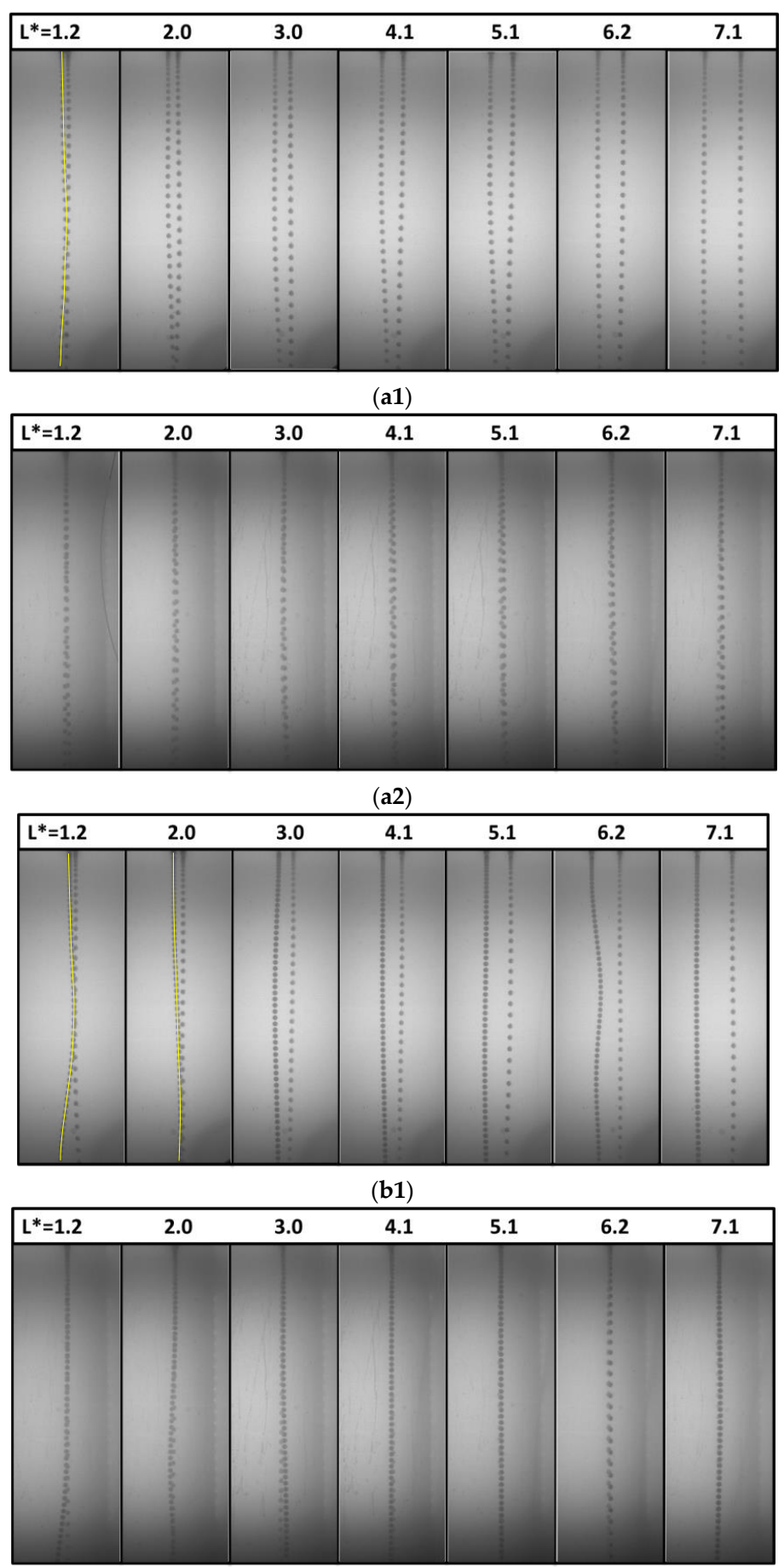

(b2)

Figure 12. Cont. 


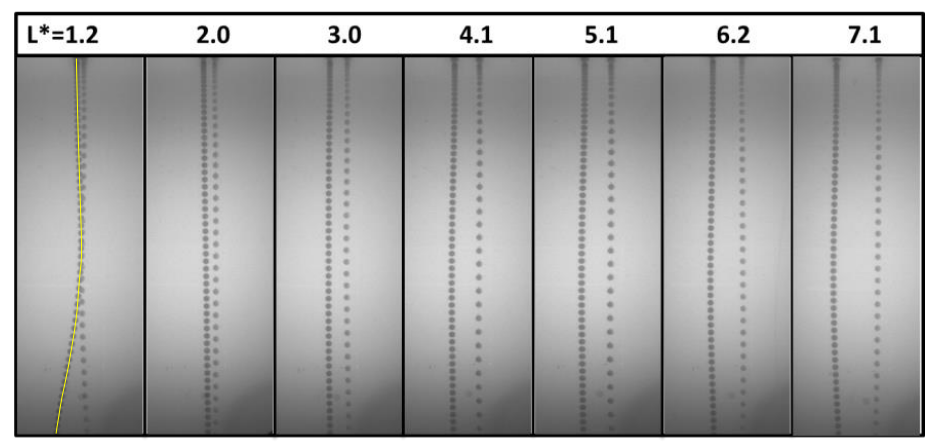

(c1)

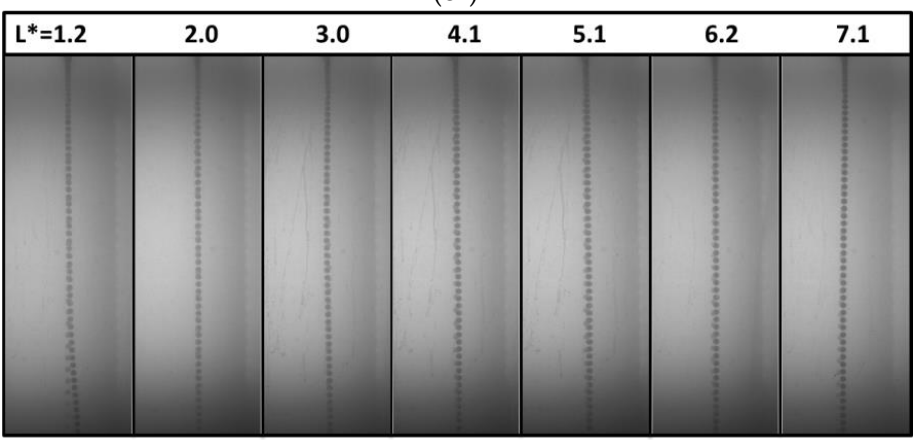

(c2)

Figure 12. The settling process of two side-by-side particles at different initial separation distance under binocular vision; (a1) $x$ - $z$ plane, Left particle: $\mathrm{ZrO}_{2}$, right particle: Stainless steel; (a2) $y$ - $z$ plane, Particle: $\mathrm{ZrO}_{2}$, Stainless steel; (b1) $x$ - $z$ plane; Left particle: $\mathrm{Si}_{3} \mathrm{~N}_{4}$; right particle: $\mathrm{ZrO}_{2}$; (b2) $y$ - $z$ plane, Particle: $\mathrm{Si}_{3} \mathrm{~N}_{4}, \mathrm{ZrO}_{2}$; (c1) $x$ - $z$ plane; Left particle: $\mathrm{Si}_{3} \mathrm{~N}_{4}$; right particle: Stainless steel; (c2) $y$ - $z$ plane, Particle: $\mathrm{Si}_{3} \mathrm{~N}_{4}$, Stainless steel.

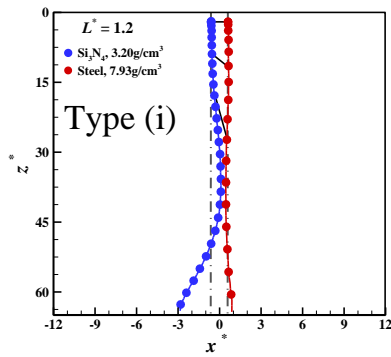

(a) Type (i)

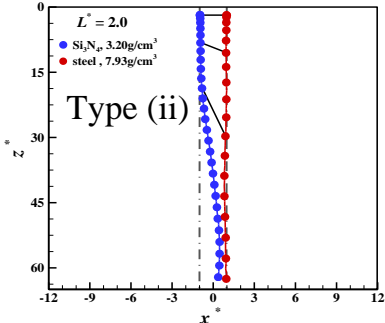

(b) Type (ii)

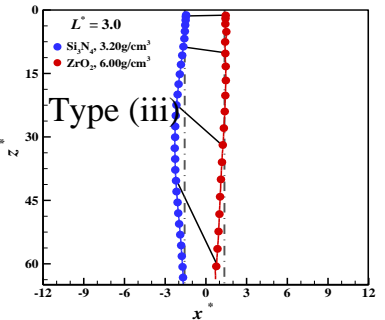

(c) Type (iii)

Figure 13. Typical settling trajectories of two particle settling side by side, with an example for each type.

In the cases of type (ii), after a time period of vertical settling of the two particles, the light particle was attracted by CRV, which is similar to the early attractive stage of type (i). The trajectories of the two particles obtained from type (ii) also exhibit a similarity to the work in which the particles tended to attract [28]. In this previous study [28], two identical stainless steel particles were released side by side; however, the two particles gradually converge until they touch each other, and then settle (one up and one down) at the same velocity. In our experiments, the density difference always causes the heavy particle to settle ahead of the light one without collision when the two particles were released side by side. Although obvious tendency of the light particle to approach the heavy one can be seen in the test section, the rebounding stage in the type (i) is not observed in type (ii), which is probably caused by our limited field of the view of the camera.

In the cases of type (iii), the light particle first slightly shifted away from its original position in the horizontal direction, and then returned to this initial position. A similar repulsive effect had been reported in the settling of two side-by-side particles of identical densities when the initial separation distance was larger than a critical value $[26,28]$. 
According to a previous deduction [28], the particle-particle repulsion can be attributed to the fluid inertia. This is a different phenomenon from the settling behaviors of two identical particles in different non-Newtonian fluids [20]. As explained above, the particle-particle attraction is mainly caused by the effects of the shear-thinning viscoelasticity properties of the fluid, hence, the first normal stress difference of the PAAm solution and the lower pressure of the CRV can drive the light particle back to its original lateral-position. It is worth mentioning that the particle-particle interaction decreased with increasing $\alpha$ or $L^{*}$, as shown in Figure 12. Particularly, when $\alpha=4.71$ and $L^{*}=7.1$, the light particle hardly affects the heavy particle, which falls as a single particle, but the disturbance of the heavy particle is still present.

\subsection{Settling Velocity}

Corresponding to the three types of settling paths in Figure 13, the snapshot of the settling velocities of a single particle and two side-by-side particles are shown in Figure 14 For path type (i), after the discharge of the particles, the settling velocities $u$ increased and the transverse velocities $v_{x}$ in $x-z$ plane remain around $0 \mathrm{~m} / \mathrm{s}$. As the light particle was dragged toward the CRV, at around $0.2 \mathrm{~s}$, the $v_{x}$ of light particle $\left(v_{x L}\right)$ started to increase and reached a maximal positive transverse-velocity of $0.031 \mathrm{~m} / \mathrm{s}$ at $t=0.422 \mathrm{~s}$. Meanwhile, the $u$ of light particle $\left(u_{L}\right)$ became higher than the terminal velocity of a single particle $(0.77 \mathrm{~m} / \mathrm{s}$ for $\mathrm{Si}_{3} \mathrm{~N}_{4}$ ), and continued to increase to the maximum value $0.866 \mathrm{~m} / \mathrm{s}$ at $t=0.641 \mathrm{~s}$. Then, the light particle rebounding from the CRV resulted in an obvious increase in the negative traverse velocity, while $u_{L}$ would fall back.

The variation of the particles settling velocities can be explained by the energy transfer. The sedimentation of a particle in a stagnant fluid can be regard as the transport between potential energy and kinetic energy accompanied by energy dissipation. For two particles settling side by side in PAAm solution, the energy dissipation due to the viscosity decreases when the light particle settles into the CRV, and more potential energy is transferred into the kinetic energy of the light particle and the fluid around it. Thus, the settling velocity of a light particle during above process will be larger than the terminal settling velocity when it settles as an isolated particle in the same fluid, as discussed above. Then the kinetic energy is transformed from the vertical direction into the horizontal direction once the lateral migration occurs, which is possibly due to the shear-thinning property of PAAm solution or the appearance of negative wake (Figure 6).

In the cases of type (ii), the $u_{L}$ can always exceed the terminal settling velocity for a single settling particle of the same density. This is possibly due to the combined effect of the lower amount of energy dissipated and the dragging of the fluid in the CRV. Moreover, as shown in Figure 14b, for mediate $L^{*}$, the particle-particle interaction tends to cause a slight fluctuation in the $v_{x L}$, which corresponds to the lateral migration of the light particle in path type (ii). With the separation distance increasing, the effects caused by the shearthinning property of PAAm solution and the negative wake act on the light particle became continuously weaker.

As for path type (iii), no significant difference in the settling velocities between the single particle and the two side-by-side particles shown in Figure 14c was found. 

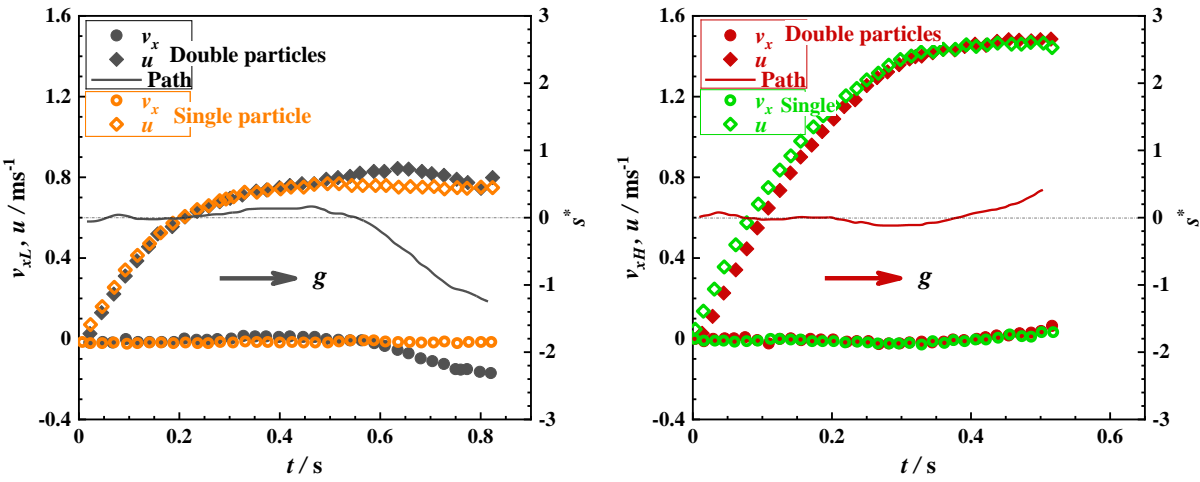

(a) Type (i), $\left(L^{*}=1.2\right.$ and $\left.\alpha=4.71\right)$
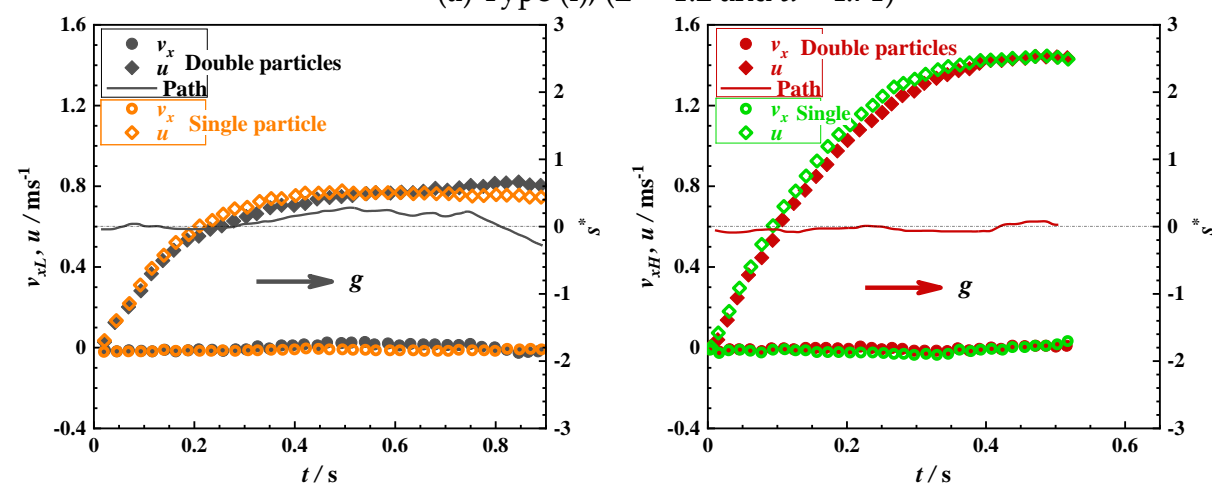

(b) Type (ii), ( $L^{*}=2$ and $\left.\alpha=4.71\right)$
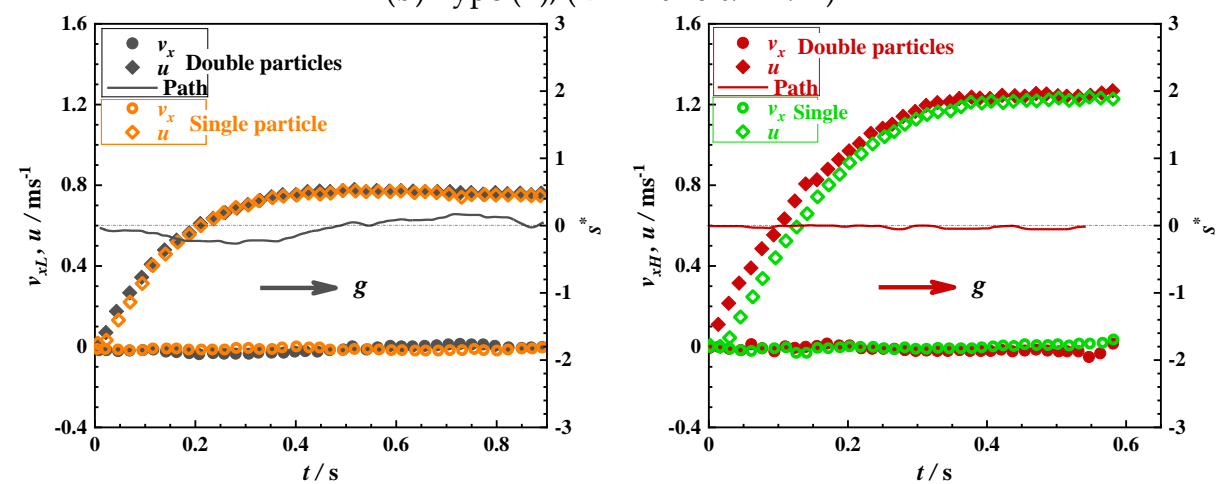

(c) Type (iii), ( $L^{*}=3$ and $\left.\alpha=2.79\right)$

Figure 14. The velocities and the trajectories of the two side-by-side settling particles at different separation distance $L^{*}$; Left: the light particle; Right: the heavy particle.

\section{Conclusions}

In this work, we studied the trajectories and velocities of two side-by-side particles of different density settling in a non-Newtonian fluid with shear thinning viscoelastic properties, as well as that for the corresponding single settling particle.

In contrast to from the three-dimensional unstable path of a single particle settling in water, the particle settling path can be regarded as a vertical line in the PAAm solution. The terminal settling velocity of the particle $\left(\mathrm{Si}_{3} \mathrm{~N}_{4}\right)$ in PAAm solution is lower than that in water. Apart from this, the terminal velocities of other particles (stainless steel and $\mathrm{ZrO}_{2}$ ) are higher than that in water, and the deviation of terminal velocity from that of the particle settling in water increases with the particle density. The reason may be that there is a critical value of $R e^{*}$, i.e., another particle with a different density, and its terminal settling velocity in the PAAm solution is consistent with that in water.

In addition to this, an experimental study on the effect of the initial separation distance on settling side-by-side particles motion at different density ratios was conducted in PAAm solution. The results show that the wake of the heavy particle, in a leading position, 
can affect the light particle settling into a non-linear path. For the heavy particle, the particle-particle interaction can also cause the transverse migration of the heavy one, and the smaller the density ratio, the greater the transverse migration. For the light particle, three basic path types were found: (i) the light particle is first attracted and then repelled by the wake of the heavy one; (ii) the light particle approaches and then largely traces within the path of the heavy one in the limited field of view; (iii) the light particle is first slightly shifted away from its original position and then returns to this initial position. In these, the light particle can be attracted or rebounded by the CRV behind the heavy particle. Meanwhile, we conclude that the $u$ of light particle became higher than the terminal velocity of a single particle, due to the existence of a corridor with a reduced viscosity and the negative wake generated by the viscoelastic property. As explained above, particle-particle attraction is mainly caused by the effects of the shear-thinning viscoelasticity properties of the PAAm solution. As discussed throughout this study, the trajectory and velocity of particle sedimentation in PAAm solution are affected by the density difference and initial spacing of the two particles and fluid properties. These findings will make contributions to the future studies and applications in this area.

Author Contributions: Conceptualization, C.T., F.B.; methodology, S.Y., M.D., R.X. and C.T.; formal analysis, X.G. (Xiaoyan Gao) and S.Y.; investigation, S.Y., M.D. and X.G. (Xianfu Ge), and R.X.; writing—original draft preparation, S.Y., C.T., X.G. (Xiaoyan Gao), and M.D.; and writingreview and editing, C.T., and F.B.; All authors have read and agreed to the published version of the manuscript.

Funding: This research received no external funding.

Acknowledgments: This work was supported by the National Natural Science Foundation of China (Grants No. 11632016, No. 11972336, No. 11872217, and No. 11972334).

Conflicts of Interest: The authors declare no conflict of interest.

\section{References}

1. Haddadi, H.; Naghsh-Nilchi, H.; Di Carlo, D. Separation of cancer cells using vortical microfluidic flows. Biomicrofluidics 2018, 12, 014112. [CrossRef] [PubMed]

2. Whitesides, G.M. The origins and the future of microfluidics. Nature 2006, 442, 368-373. [CrossRef]

3. Xuan, X.; Zhu, J.; Church, C. Particle focusing in microfluidic devices. Microfluid. Nanofluidics 2010, 9, 1-16. [CrossRef]

4. Fernando, H.; Lee, S.; Anderson, J.; Princevac, M.; Pardyjak, E.; Grossman-Clarke, S. Urban fluid mechanics: Air circulation and contaminant dispersion in cities. Environ. Fluid Mech. 2001, 1, 107-164. [CrossRef]

5. Clift, R.; Grace, J.R.; Weber, M.E. Bubbles, Drops, and Particles; Dover Publications, Inc.: Mineola, NY, USA, 2005.

6. Magnaudet, J.J. The forces acting on bubbles and rigid particles. In Proceedings of the ASME Fluids Engineering Division Summer Meeting, FEDSM, Vancouver, BC, Canada, 22-26 June 1997; pp. 22-26.

7. Zenit, R.; Feng, J. Hydrodynamic interactions among bubbles, drops, and particles in non-Newtonian liquids. Annu. Rev. Fluid 2018, 50, 505-534. [CrossRef]

8. Horowitz, M.; Williamson, C. The effect of Reynolds number on the dynamics and wakes of freely rising and falling spheres. J. Fluid Mech. 2010, 651, 251. [CrossRef]

9. Jenny, M.; Dusek, J.; Bouchet, G. Instabilities and transition of a sphere falling or ascending freely in a Newtonian fluid. J. Fluid Mech. 2004, 508, 201. [CrossRef]

10. $\mathrm{Wu}, \mathrm{J} . ;$ Manasseh, R. Dynamics of dual-particles settling under gravity. Int. J. Multiph. Flow 1998, 24, 1343-1358. [CrossRef]

11. Nie, D.; Lin, J. Simulation of sedimentation of two spheres with different densities in a square tube. J. Fluid Mech. 2020, 896. [CrossRef]

12. Apte, S.V.; Martin, M.; Patankar, N.A. A numerical method for fully resolved simulation (FRS) of rigid particle-flow interactions in complex flows. J. Comput. Phys. 2009, 228, 2712-2738. [CrossRef]

13. Wacholder, E.; Sather, N. The hydrodynamic interaction of two unequal spheres moving under gravity through quiescent viscous fluid. J. Fluid Mech. 1974, 65, 417-437. [CrossRef]

14. D'Avino, G.; Maffettone, P.L. Particle dynamics in viscoelastic liquids. J. Non Newton. Fluid Mech. 2015, 215, 80-104. [CrossRef]

15. Kumar, G.; Natale, G. Settling dynamics of two spheres in a suspension of Brownian rods. Phys. Fluids 2019, 31, 073104. [CrossRef]

16. Malhotra, S.; Sharma, M.M. Settling of spherical particles in unbounded and confined surfactant-based shear thinning viscoelastic fluids: An experimental study. Chem. Eng. Sci. 2012, 84, 646-655. [CrossRef]

17. Uchiyama, S.; Noda, M.; Krayukhina, E. Sedimentation velocity analytical ultracentrifugation for characterization of therapeutic antibodies. Biophys. Rev. 2018, 10, 259-269. [CrossRef] [PubMed] 
18. Heyder, J. Deposition of inhaled particles in the human respiratory tract and consequences for regional targeting in respiratory drug delivery. Proc. Am. Thorac. Soc. 2004, 1, 315-320. [CrossRef] [PubMed]

19. Hu, X.; Lin, J.; Chen, D.; Ku, X. Influence of non-Newtonian power law rheology on inertial migration of particles in channel flow. Biomicrofluidics 2020, 14, 014105. [CrossRef]

20. Sulaymon, A.H.; Wilson, C.A.; Alwared, A.I. An experimental investigation of the settling behavior of two spheres in a power law fluid. J. Non Newton. Fluid Mech. 2013, 192, 29-36. [CrossRef]

21. Hsu, J.-P.; Shie, C.-F.; Tseng, S. Sedimentation of a cylindrical particle in a Carreau fluid. J. Colloid Interface Sci. 2005, 286, 392-399. [CrossRef] [PubMed]

22. Gheissary, G.; Van Den Brule, B. Unexpected phenomena observed in particle settling in non-Newtonian media. J. Non Newton. Fluid Mech. 1996, 67, 1-18. [CrossRef]

23. Solomon, M.; Muller, S. Flow past a sphere in polystyrene-based Boger fluids: The effect on the drag coefficient of finite extensibility, solvent quality and polymer molecular weight. J. Non Newton. Fluid Mech. 1996, 62, 81-94. [CrossRef]

24. Goyal, N.; Derksen, J. Direct simulations of spherical particles sedimenting in viscoelastic fluids. J. Non Newton. Fluid Mech. 2012, 183, 1-13. [CrossRef]

25. Riddle, M.J.; Narvaez, C.; Bird, R.B. Interactions between two spheres falling along their line of centers in a viscoelastic fluid. J. Non Newton. Fluid Mech. 1977, 2, 23-35. [CrossRef]

26. Joseph, D.; Liu, Y.; Poletto, M.; Feng, J. Aggregation and dispersion of spheres falling in viscoelastic liquids. J. Non Newton. Fluid Mech. 1994, 54, 45-86. [CrossRef]

27. Bot, E.; Hulsen, M.; Van den Brule, B. The motion of two spheres falling along their line of centres in a Boger fluid. J. Non Newton. Fluid Mech. 1998, 79, 191-212. [CrossRef]

28. Gumulya, M.; Horsley, R.; Pareek, V.; Lichti, D. The effects of fluid viscoelasticity on the settling behaviour of horizontally aligned spheres. Chem. Eng. Sci. 2011, 66, 5822-5831. [CrossRef]

29. Pan, T.-W.; Chiu, S.-H.; Glowinski, R. Numerical study of two balls settling in viscoelastic fluids from an initial vertical configuration. Phys. Fluids 2019, 31, 123104.

30. Freire, D.; Sarasúa, L.; Vernet, A.; Varela, S.; Usera, G.; Cabeza, C.; Martí, A. Separation regimes of two spheres falling in shear-thinning viscoelastic fluids. Phys. Rev. Fluids 2019, 4, 023302. [CrossRef]

31. Eisenberg, D.; Klink, I.; Phillips, R. Axisymmetric sedimentation of spherical particles in a viscoelastic fluid: Sphere-wall and sphere-sphere interactions. J. Rheol. 2013, 57, 857-880. [CrossRef]

32. Liu, C.; Shao, H.; Chen, F.; Zheng, H. Rheological properties of concentrated aqueous injectable calcium phosphate cement slurry. Biomaterials 2006, 27, 5003-5013. [CrossRef]

33. Arigo, M.T.; McKinley, G.H. An experimental investigation of negative wakes behind spheres settling in a shear-thinning viscoelastic fluid. Rheol. Acta 1998, 37, 307-327. [CrossRef]

34. Harlen, O.G. The negative wake behind a sphere sedimenting through a viscoelastic fluid. J. Non Newton. Fluid Mech. 2002, 108, 411-430. [CrossRef]

35. Nie, D.; Lin, J.; Gao, Q. Settling behavior of two particles with different densities in a vertical channel. Comput. Fluids 2017, 156, 353-367. [CrossRef]

36. Chhabra, R.P. Bubbles, Drops, and Particles in Non-Newtonian Fluids; CRC Press: Boca Raton, FL, USA, 2006.

37. Reynolds, P.; Jones, T. An experimental study of the settling velocities of single particles in non-Newtonian fluids. Int. J. Mineral. Process. 1989, 25, 47-77. [CrossRef]

38. Acharya, A.; Mashelkar, R.; Ulbrecht, J. Flow of inelastic and viscoelastic fluids past a sphere. Rheol. Acta 1976, 15, 454-470. [CrossRef]

39. Lockyer, M.; Davies, J.; Jones, T. The importance of rheology in the determination of the carrying capacity of oil-drilling fluids. In Rheology; Springer: Berlin, Germany, 1980; pp. 127-132.

40. Xu, R.; Tu, C.; Bao, F.; Yin, Z. Study on the dynamic characteristics of two side-by-side particles of different density settling in water. J. China Univ. Metrol. 2020, 31, 168-176.

41. Fraggedakis, D.; Dimakopoulos, Y.; Tsamopoulos, J. Yielding the yield-stress analysis: A study focused on the effects of elasticity on the settling of a single spherical particle in simple yield-stress fluids. Soft Matter 2016, 12, 5378-5401. [CrossRef] [PubMed]

42. Liu, M.-1. Numerical simulation of particle sedimentation in 3D rectangular channel. Appl. Math. Mech. 2011, 32, 1147. [CrossRef] 